\title{
Endogenous Formation of Partnerships with Moral Hazard "
}

\author{
María Paz Espinosa* and Inés Macho-Stadler**
}

First version: September 15, 1998

This version: February 29, 2000

WORKING PAPER 448.00

\begin{abstract}
We analyze the formation of partnerships as a sequential game with moral hazard within coalitions; once formed, partnerships compete a la Cournot in the marketplace. When moral hazard within coalitions is very severe, no partnership will form. However, we show that when moral hazard is not too severe the coalition structure will be either similar to or more concentrated than it is without moral hazard. Concerning industry profits, without moral hazard too many coalitions are formed in equilibrium as compared to the efficient outcome, but moral hazard may be responsible for an inefficiency of opposite sign.
\end{abstract}

Keywords: endogenous coalition formation, moral hazard, partnerships.

\# We are thankful to David Pérez-Castrillo and seminar participants at the ASSET meeting (Tel Aviv), XXII BWED, CentER at Tilburg University, and the Workshop of Coalition Formation (Barcelona) for many useful comments. Financial support from UPV (035.321-HB021/96), Gobieerno Vasco (PI-1998-86), DGES (PB 97-0603) and Generalitat (SGR 98-62) is gratefully acknowledged. Any errors are our own.

Universidad del País Vasco. Departamento de Fundamentos del Análisis Económico. Avenida Lehendakari Aguirre 83. E-48015 Bilbao (Spain). E-mail: jepesalp@bs.ehu.es

** Universitat Autònoma de Barcelona. CODE \& Departament d'Economia i d'Historia Econòmica. E-08193 Bellaterra (Spain).E-mail: Ines.Macho@uab.es 


\section{1.- Introduction}

Game theory has provided a framework to analyze market structure determination; the incentives of firms to merge or to form associations can be usefully analyzed in games of endogenous coalition formation with spillovers. In this literature, coalition formation is analyzed as a two-stage game. In the first stage, players form groups and this process of team formation is formalized as a non-cooperative game (either simultaneous or sequential). ${ }^{1}$ Once the groups are formed, the members of each group are committed to maximize the group's objective function in the second stage. This family of games defines two levels of interaction among the players: first, within each coalition and, second, among the coalitions. While the second level of interaction is formalized in a non-cooperative way, the first one assumes complete cooperation among the coalition members.

Cooperation within a coalition can emerge if the coalition members have mechanisms that allow them to commit to their behavior or all the relevant information is verifiable and contracts are complete. However, one can imagine situations where full cooperation among non-cooperative players is not possible. In other words, the abovee approach ignores that in some circumstances players in a coalition may retain some decision-making power over the strategic variables in the second stage, and they may not share exactly the same interests. The problem of opportunistic behavior within coalitions can arise in games considering the determination of market concentration and size of partnerships, when partners can free-ride on their colleagues. It can also be present in situations like the formation of custom unions

1 Different rules for coalition formation have been used in literature. Bloch $(1995,1996)$, Ray and Vohra (1999) and Montero (1999) examine an infinite-horizon game where coalitions form when all potential members agree. The former papers assume a fixed rule of order; in the latter, the player who rejects an offer does not automatically become the next proposer. Ray and Vohra (1997) consider a model where deviations can only serve to make the existing coalition structure 
or international tax agreements, where countries agree to form a coalition but keep some room to take decisions departing from the coalition's global objective. OPEP may be cited as an example.

Within a coalition, a problem of moral hazard arises when partners decide individually on the second stage strategic variables. ${ }^{2}$ As Holmström (1982) proved, moral hazard in teams leads to inefficiencies for any (balanced budget) sharing rule since team members do not receive the total revenue from their effort.

The aim of this paper is to analyze the equilibrium coalition structures and the efficiency of the equilibrium outcome in a model with moral hazard within coalitions, under the assumption that productive coalitions compete à la Cournot and use an egalitarian profitsharing rule. ${ }^{3}$ In this context, a coalition is a set of players who agree to share output (equally) but behave throughout the relationship in a non-cooperative way. To our knowledge, this is the first paper that models this behavior.

To highlight the market competition and moral hazard effects we shall ignore any team economies; adding team economies would partly obscure the moral hazard problem: big coalitions have more severe incentive problems but (with team economies) they would be more efficient. ${ }^{4}$ We focus on the interaction between moral hazard within the partnership, product market competition, and the emergence of different coalition structures. Our concern is the determination of the number of partnerships active in the market and their

finer. Yi and Shin (1995) and Belleflamme (2000) study games in which non-members can join a coalition without the permission of the existing members.

2 See, for example, Macho-Stadler and Pérez-Castrillo (1997) for more details on incentives and contract design under moral hazard.

3 In this aspect, we follow Farrell and Scotchmer (1988), who define a partnership as a coalition that divides its output equally.

4 Bloch (1995) and Belleflamme (2000) consider the case where firms form associations in order to decrease their costs. This aspect is also taken into account in the existing theory of partnerships, where partners get together to exploit economies of scale (see, for example, Farrell and Scotchmer, 1988; and Sherstyuk, 1998). In this partnership literature there is no market competition, while in our approach partnerships form to get a higher market concentration, and thus higher prices and profits, and they form despite the fact that there is a moral hazard problem. 
size, taking into account the demand and cost conditions. In the absence of moral hazard, two forces affect the final market structure. On the one hand, decreasing the number of firms implies benefits related to a lower degree of competition in the market, but, on the other hand, a player may increase her profits by leaving a big coalition and setting up a firm by herself. Therefore, the process of partnership formation may exhibit inefficiencies (from the industry point of view) due to a free-riding problem among coalitions. Hence, too many firms are formed in equilibrium (this is a well known result; see Salant, Switcher and Reynolds, 1984, Bloch, 1996, and Yi, 1997, among others).

The presence of moral hazard within coalitions adds a new effect to the previous ones: building up a large coalition involves efficiency losses as the incentive problems are more severe in larger partnerships. In the extreme case of a very severe moral hazard problem, we show that the equilibrium market structure is very fragmented, with each player setting up their own firm. However, if moral hazard is not so severe, not only we will not find a very fragmented industry, but the number of coalitions will be lower than or equal to the number that emerges without moral hazard. For intermediate levels of moral hazard, the number of partnerships is strictly lower than without moral hazard. The reason behind this result is that moral hazard makes it more difficult to free ride on other players, precisely because under moral hazard other players are less willing to form large coalitions.

As far as the efficiency of the equilibrium coalition formation is concerned, without moral hazard the grand coalition is efficient (it maximizes industry profits) but it is not an equilibrium market structure. We show that under moral hazard this may be no longer the case. This effect is partly due to the fact that moral hazard also affects the efficiency of the grand coalition; when moral hazard is high enough, a monopoly is not efficient and other market structures may yield higher industry profits.

The paper is organized as follows. Section 2 describes the sequential model of partnership formation, which follows Bloch (1996)'s sequential games of coalition formation, and derives the utility function of each partner as a function of market structure. In Section 3, we characterize the equilibrium outcome. Section 4 is devoted to a discussion on the robustness of our analysis. Section 5 deals with the efficiency of the equilibrium coalition configuration. Section 6 concludes. 


\section{2.- The model}

We study the interaction among (ex-ante identical) partners as a game in two stages. In the first stage, partners group into firms through a sequential process, and in the second stage of the game firms compete à la Cournot with a homogeneous product. First, we describe the process of constitution of partnerships. Then we analyze the market competition stage with a moral hazard problem inside firms. We assume that gross profits are shared equally among the partners but they decide individually how much effort to exert; this effort is not verifiable and cannot be contracted upon. The implication is that partnerships competing in the market will not necessarily be profit maximizing firms. In the last part of this section we derive the payoff function for each partner as a function of market structure.

\section{1.- The partnership formation game}

The total number of partnerships competing in the market is determined endogenously. There is a fixed number of ex-ante identical partners, $n$, and they have to decide, before market competition, how many partnerships they will set up. We follow Bloch (1996)'s approach and assume partnerships are formed sequentially.

Bloch (1996) and Ray and Vohra (1999) analyze an infinite-horizon sequential game that we will denote by $\Gamma$, in which a coalition forms if and only if all potential members agree to form the coalition. There is a rule of order $\rho$ (an ordering of the players) on the set of partners, $N$, that determines the order of moves in the sequential game. Partner 1 , according to the rule of order, makes an offer to other agents to form a coalition of partners $T \subset N$, to which she belongs. Each prospective member in $T$ responds to the offer in the order determined by $\rho$. If all members in $T$ accept the offer of partner 1 , the partnership is formed and partners in $T$ leave the game. Then, the first partner in $N \backslash T$, according to the rule of order, starts the game (with $N \backslash T$ players) by making a partnership proposal, and the game continues. If any member in $T$ rejects the offer made by partner 1 , that partner makes a counteroffer and proposes the formation of a partnership $S \subset N$, to which he belongs. The game continues until all players have left the game. The outcome of this game is a market structure, that is, a partition of the set of partners, $P=\left\{T_{1}, T_{2}, \ldots, T_{r}\right\}$, representing all the firms formed. 
Bloch (1996) shows that when players are ex-ante symmetric the partitions generated by the infinite-horizon sequential game (i.e., the stationary symmetric subgame perfect equilibrium coalition structures) coincide with the partitions generated by the following choice-of-sizes game. ${ }^{5}$ The first partner, according to the rule of order, announces an integer $s_{1}$, interpreted as the size of the firm, and the first $s_{1}$ players form a coalition. Then partner $s_{1}+1$ announces an integer $s_{2}$, and the next $s_{2}$ players form a coalition, and the game proceeds until $s_{1}+s_{2}+s_{3}+\ldots=n$. We shall solve the choice-of-sizes game to determine the equilibrium market structures brought about by partners' behavior under moral hazard.

\section{2.- The Moral Hazard Problem}

Each firm $A$ is an association of $n_{A}$ ex-ante identical partners who own and manage the firm. Partners produce a homogeneous output and for simplicity we assume the only production costs come from the effort exerted to produce. Output is normalized to be equal to the productive effort so that the production function of firm $A$ is:

$$
q_{A}=\sum_{i=1}^{n_{A}} e_{i A}
$$

where $q_{A}$ is output of firm $A$ and $e_{i A}$ is effort exerted by partner $i$ in firm $A$.

Assume that there are $r$ associations of partners competing in the market: 1, 2, .., $r$. Gross profits (ignoring the costs of partners' effort) for each firm $A, A \in\{1,2, \ldots, r\}$ are $\Pi_{A}\left(\sum_{i=1}^{n_{1}} e_{i 1}, \sum_{i=1}^{n_{2}} e_{i 2}, \ldots, \sum_{i=1}^{n_{r}} e_{i r}\right)$. Effort within the firm is not verifiable so that the output (or revenue) due to each partner's effort cannot be identified. Partners are assumed to share the

5 A property of non-decreasing equilibrium payoffs is needed for this result to hold; we discuss this property after presenting the results. For more details on the infinite-horizon game and the proof of this result see Bloch (1996). 
gross profits of the firm equally. ${ }^{6}$ The level of gross profits is verifiable and thus partners can commit to an equal sharing rule in the first stage.

To compute the net profits of a firm we need to define the cost of a partner's effort, which is assumed to be independent of the firm size, $C\left(e_{i}\right)$. Thus, net profits for firm $A$ are:

$$
\Pi_{A}\left(\sum_{i=1}^{n_{1}} e_{i 1}, \sum_{i=1}^{n_{2}} e_{i 2}, \ldots, \sum_{i=1}^{n_{r}} e_{i r}\right)-\sum_{i=1}^{n_{A}} C\left(e_{i}\right)
$$

The interaction among partners in the second stage is modeled as a non-cooperative game in which the level of effort $e_{i A}$ is decided by each partner $i$ and is non-verifiable. Thus, each partner $i$ in firm $A$ decides her level of effort to maximize her own payoff function:

$$
\frac{1}{n_{A}} \Pi\left(\sum_{i=1}^{n 1} e_{i 1} \sum_{i=1}^{n 2} e_{i 2}, \ldots, \sum_{i=1}^{n r} e_{i r}\right)-C\left(e_{i A}\right) .
$$

Due to the moral hazard problem, this behavior implies that firms do not maximize profits.

\section{3.- The Payoff Function}

We shall assume that, after the process of constitution of partnerships, firms compete in the market for a homogeneous product with linear market demand, $p=a-Q$, where $Q$ is aggregate output. The production stage is formalized as Cournot competition. Gross profits for firm $A$ are:

$$
\Pi_{A}\left(\sum_{i=1}^{n 1} e_{i 1}, \ldots, \sum_{i=1}^{n r} e_{i r}\right)=\left[a-\left(\sum_{i=1}^{n 1} e_{i 1}+\ldots+\sum_{i=1}^{n r} e_{i r}\right)\right] \sum_{i=1}^{n_{A}} e_{i A}
$$

6 When partners are ex-ante identical, the equal-sharing rule is a natural division of payoffs. Ray and Vohra (1999), in an infinite-horizon model, make the sharing rule endogenous and show that equal sharing is an equilibrium phenomenon. Note however that in their framework the sharing rule will influence the decision of whether (or not) to enter a coalition, while in our model the sharing rule will also affect the incentives of the members of a coalition. Limited liability arguments or commitment problems can lead to equal sharing rules in our framework. 
The cost of exerting effort for partner $i$ is: $C\left(e_{i}\right)=c e_{i}$, with $c<a$. The situation where there is no moral hazard within the firm will be referred to as the benchmark case.

2.3.1.- The benchmark case. Assume that there is no moral hazard on partners' decisions. Partners' effort is verifiable, so that the cooperative level can be implemented within a coalition. Taking into account that partners are symmetric, the best response to rival firms' effort can be expressed as:

$$
e_{A}=\frac{a-\sum_{j \neq A} e_{j} n_{j}-c}{2 n_{A}},
$$

for $A \in\{1, \ldots, r\}$, where $e_{j}$ denotes the (symmetric) effort level by each partner in firm $j$. Solving the system, from the optimal effort level, we have that:

$$
q_{A}^{*}=n_{A} e_{A}^{*}=\frac{a-c}{r+1} .
$$

Note that $q_{A}^{*}$ is independent of the size of the partnership $n_{A}$, and the number of members $n$, and depends only on the number of firms $r$. This is because there is an optimal output level for each firm as a function of market demand and cost conditions, and each firm just divides the burden of producing the optimal output equally among its members. The payoff function for each partner of firm $A$ in the benchmark case is:

$$
\pi_{i}\left(r, n_{A}\right)=\frac{(a-c)^{2}}{(r+1)^{2} n_{A}} .
$$

2.3.2.- The Payoff Function under Moral Hazard. Due to the moral hazard problem, firms do not maximize profits. Rather, each partner decides how much effort she will exert to maximize her own objective function. Each partner in firm $A$ will maximize her objective function on the effort level $e_{i A}$. From the first-order conditions, we obtain:

$$
e_{i A}=\frac{a-c n_{A}-\sum_{j \neq A} e_{j} n_{j}-2 \sum_{k \neq i} e_{k A}}{2}
$$


for $A \in\{1, \ldots, r\}, i \in A$, and where $e_{j}$ denotes the (symmetric) effort level by each partner in firm $j$ for $j \neq A$. Efforts are strategic substitutes: if other players (from inside or outside the relationship) increased their effort level, the best response for a member of firm $A$ would be to decrease her own. Solving the system formed by the first order conditions of the $n$ partners, we obtain the optimal effort for each partner in firm $A$, and the optimal output for firm $A$ :

$$
q_{A}=n_{A} e_{A}=\frac{a+c\left[n-(r+1) n_{A}\right]}{r+1} .
$$

Given the assumption of linear demand and cost, the output level $q_{A}$ does not depend on the distribution of sizes of all the coalitions in the market, but only on $n_{A}, n$, and $r$. The optimal effort for a partner is decreasing in $n_{A}$, since the higher $n_{A}$ the higher the intensity of the moral hazard problem; it is increasing in $n$ since this variable is a measure of the aggregate output (the relative size) of the other coalitions. The effect of cost $c$ is positive (or negative) if the coalition is small (big) as compared to the others. Note that, in contrast with the benchmark case, the output of a firm depends on its size.

The distortion introduced by the moral hazard problem can be seen by comparing expression (3) to the effort in the benchmark case, given by expression (2):

$$
e_{A}^{*}-e_{A}>0 \Leftrightarrow n_{A}>\frac{n+1}{r+1}
$$

Since $\frac{n+1}{r+1}$ is approximately the average size of coalitions, for coalitions of a size higher than the average, moral hazard causes a distortion in the usual direction, that is, it decreases output. However, for coalitions of a size lower than the average the "best response effect" (or strategic effect) dominates the partners' incentives to take an opportunistic decision on their effort. For these relatively small coalitions, the fact that rivals have a more serious moral hazard problem than theirs induces them to expand output. With market competition, what is important is how serious a firm's moral hazard problem is as compared to the average moral hazard problem of its rivals, since this comparison determines the relationship between own and rivals' production cost. 
We can also compare total output in the benchmark case and under moral hazard, for a given coalitional structure. From the expressions:

$$
\sum_{j} e_{j}^{*} n_{j}=\frac{r a-r c}{r+1}, \quad \text { and } \quad \sum_{j} e_{j} n_{j}=\frac{r a-c n}{r+1}
$$

the intuitive result that total output is lower under moral hazard can immediatly be checked. Moral hazard reduces production, even if the smaller coalitions produce more output than in the benchmark case. The strategic effect does not offset the overall distortion associated to moral hazard. From the foregoing expressions, it can also be checked that this distortion associated to moral hazard within coalitions is decreasing in $r$ and increasing in $n$ and $c$. Total output in the benchmark case depends only on the number of coalitions while with moral hazard it also depends on the total number of players, which summarizes (in this linear model) the overall inefficiency due to the size of the coalitions; note that the distorsion introduced by moral hazard disappears when $r=n$.

Substituting the equilibrium efforts, the payoff for a partner can be expressed as: ${ }^{7}$

$$
\pi_{i}\left(r, n_{A}\right)=\frac{1}{n_{A}}\left(\frac{a+c n}{r+1}-c\right)\left(\frac{a+c n}{r+1}-c n_{A}\right)
$$

Given the parameters of the model $(n, a, c)$, payoffs depend on $n_{A}$ and $r$. This payoff function for each of the $n$ players, derived from competition in the product market, gives the payoff to each partner as a function of the market structure, and it will determine the outcome of the partnerships' formation game.

\section{3.- On the equilibria of the sequential partnership formation game}

We denote by $\Delta(n, a, c)$ the sequential game of size selection with $n$ players, demand parameter $a$, unit cost $c$, and payoffs given by (4). We recall the description of the choice-

\footnotetext{
${ }^{7}$ If demand and cost were non-linear, profits (with or without moral hazard) would depend also on the sizes of other coalitions.
} 
of-sizes game. Player 1 starts the game and chooses an integer $s_{1}$ in the set $\{1,2, \ldots, n\}$. Player $s_{1}+1$ then moves and chooses an integer $s_{2}$ in the set $\left\{1,2, \ldots, n-s_{1}\right\}$, and so on. The game continues until the sequence of integers $\left(s_{1}, s_{2}, . ., s_{r}\right)$ satisfies $\sum_{i=1}^{r} s_{i}=n$.

Any subgame of $\Delta(n, a, c)$ such that, $h$ coalitions have been formed already, $h \leq n-1$, and the number of players who have not yet been assigned to any partnership is $x, x \leq n-h$, will be denoted by the pair $(h, x){ }^{8}$ In subgame $(h, x)$, the player who makes the first announcement is player $(n-x+1)$ and she chooses $s_{h+1}$ in the set $\{1,2, \ldots, x\}$.

A first result states the intuitive outcome that if moral hazard is very severe, which in our model can be interpreted as $c$ big as compared to $a$, then no player will join a coalition in a subgame perfect equilibrium (SPE). That is, partnerships are not sustainable in equilibrium.

Proposition 1.- If the degree of moral hazard is high enough, $\frac{a}{c}<\frac{n}{n-1}$, the unique SPE partition is all singletons.

Proof.- See Appendix.

The next two lemmas state technical results that will help us to analyze the outcome of the choice-of-sizes game.

Lemma 1.- Consider that player $(n-x+1)$, deciding in subgame $(h, x)$, has to choose size $s$, under the assumption that the remaining $(x-s)$ players will announce size 1 when it is their turn. Then, her optimal choice is either $s=1$, or $s=x$. Formally:

$$
\max _{1 \leq s \leq x} \pi_{n-x+1}(h+1+x-s, s)
$$

8 In our model, at any time in the game, $h$ and $x$ are the only relevant variables. More precisely, $(h, x)$ is a set of subgames with the same $h$, the same $x$ and the same player $(n-x+1)$ deciding (although other aspects of the history may be different). All these subgames are equivalent as far as the decisions of player $(n-x+1)$ and subsequent players (according to the rule of order $\rho$ ) are concerned. 
always has a corner solution.

Proof: See Appendix.

In order to identify the corner solution of the maximization problem presented in Lemma 1, define the function $g(h, x)$ as:

$$
g(h, x) \equiv \pi_{n-x+1}(h+1, x)-\pi_{n-x+1}(h+x, 1)
$$

where $\pi_{n-x+1}$ is the function defined in (4). When $g(h, x)$ is positive, the $x$ players who have not entered any partnership yet are better off joining a partnership than they are as singletons. When $g(h, x)$ is negative, however, they would rather form $x$ firms than a single firm.

Lemma 2.- (i) For any subgame $(h, x)$ of $\Delta(n, a, c)$ :

$$
\operatorname{sign}[g(h, x)]=\operatorname{sign}\left[(a+c n)\left(x-(h+1)^{2}\right)-c(h+2)\left(x^{2}-(h+1)^{2}\right)\right] .
$$

(ii) A necessary condition for $g(h, x) \geq 0$ is that:

$$
x-(h+1)^{2}>0 .
$$

(iii) In a subgame $(h, x)$ such that $x \leq(h+1)^{2}$, it is the case that $g\left(h^{\prime}, x^{\prime}\right)<0$ for any subgame $\left(h^{\prime}, x^{\prime}\right)$ of $(h, x)$.

Proof: See Appendix.

Lemma 2 (i) rewrites the condition on the sign of $g(h, x)$ in terms of the parameters of the game. Part (ii) gives a necessary condition for the sign of this function to be positive, i.e. player $(n-x+1)$ prefers forming a coalition with all the remaining players than a situation in which all of them remain as singletons. Part (iii) says that if $x \leq(h+1)^{2}$, then it is better for player $(n-x+1)$ in subgame $(h, x)$, and also for all the remaining players in the game, staying as singletons $(s=1)$ than joining all the subsequent players.

We now analyze the outcome of the game $\Delta(n, a, c)$. More precisely, we are going to provide necessary conditions for coalition structures to be sustainable in a SPE of the game. For convenience, we shall consider that the player who is indifferent between 
forming a coalition with the remaining players or breaking apart and inducing all the remaining players to form singletons, will choose to form the coalition. The following proposition starts the analysis by providing simple necessary conditions for the two extreme coalition structures, monopoly and all singletons, to emerge.

Proposition 2.- (i) A necessary condition for monopoly to be an equilibrium coalition configuration is $g(0, n) \geq 0$, i.e., $\frac{a}{c} \geq n+2$.

(ii) A necessary condition for all singletons to be an equilibrium coalition configuration is $g(0, n)<0$, i.e., $\frac{a}{c}<n+2$.

Proof.- (i) A monopoly forms only if $s_{1}=n$. We claim that this cannot be an equilibrium if $g(0, n)<0$, since $s^{\prime}{ }_{1}=1$ is a better strategy for player 1 . Indeed, after $s_{1}^{\prime}=1$, the worst that can happen to player 1 is that the other players also choose $s_{i}=1$ and this is preferred by 1 to monopoly because $g(0, n)<0$. Also, using Lemma 2 (i), it is easy to check that $\frac{a}{c}<n+$ 2 is equivalent to $g(0, n)<0$.

(ii) $\frac{a}{c} \geq n+2$ is equivalent to $g(0, n) \geq 0$. In this case, player 1 would rather form a monopoly by choosing $s_{1}=n$ than play a strategy that will lead to an $n$-poly. $\quad$ Q.E.D.

In the following proposition we provide necessary conditions for other coalition structures to emerge. The argument goes as follows. For an $r$-poly to be an equilibrium structure, it is necessary that at least in one continuation of the game, once $(r-1)$ coalitions have been formed, the deciding responsible player should join all the others. The proposition reproduces this argument in terms of the parameters of the model.

Proposition 3.- A necessary condition for an $r$-poly to be an equilibrium coalition structure, with $n>r \geq 2$, is:

$\frac{a}{c} \geq \frac{(r+1)(n+1)(n-2 r+1)}{\left(n+1-r-r^{2}\right)}-n, \quad$ when $n<r\left(1+r+\sqrt{r^{2}-1}\right)-1$, or

$\frac{a}{c} \geq 2 r(r+1)\left(r+\sqrt{r^{2}-1}\right)-n, \quad$ when $n \geq r\left(1+r+\sqrt{r^{2}-1}\right)-1$. 
The above condition is also necessary for an $\bar{r}$-poly to be an equilibrium outcome, for any $\bar{r}$, with $n>\bar{r} \geq r$.

Proof.- The proof is merely outlined here. See the complete proof in the Appendix. It proceeds in several steps. We first prove that, for $r<n$, a necessary condition for an $r$-poly to be an equilibrium coalition configuration is that $g(r-1, x) \geq 0$, for some $x \leq n-r+1$. Then, we rewrite this necessary condition in terms of the parameters of the model. Finally, we prove that the necessary condition for an $r$-poly to emerge must also be satisfied for an $\bar{r}$-poly to emerge, with $\bar{r} \neq n$ and $\bar{r} \geq r \geq 2$. Formally:

$$
\left.\left.\left.\left.[g(r-1, x) \leq 0, \forall x \in] r^{2}, n-r+1\right]\right] \Rightarrow[g(r, x)<0, \forall x \in](r+1)^{2}, n-r\right]\right] . \quad \text { Q.E.D. }
$$

To see if the number of firms in an equilibrium coalition configuration can be very high, lets us consider the maximum possible number of partnership, excluding the possibility that all players stay alone. In other words, we derive an upper-bound to the number of coalitions that can possibly constitute an equilibrium coalition structure, different from all singletons.

Proposition 4.- If an $r$-poly, with $r<n$, is an equilibrium coalition structure, then $r \leq$ $r^{\max }(n)$, where: $r^{\max }(n)=\frac{-1+\sqrt{5+4 n}}{2}$.

Proof.- For an $r$-poly to fulfill the necessary condition derived in Proposition 3, there must exist a non-degenerate interval with $r^{2}<x \leq n-r+1$. Hence, the maximum $r$ that can be stable is the $r^{\max }$ such that $\left(r^{\max }\right)^{2}=n-r^{\max }+1$, i.e., $r^{\max }(n)=\frac{-1+\sqrt{5+4 n}}{2}$. Q.E.D.

First of all, note that the upper-bound $r^{\max }(n)$ defined in Proposition 4 coincides with the number of coalitions in the equilibrium structure when there is no moral hazard. Bloch (1996) shows that without moral hazard the equilibrium coalition configuration is such that all the first players choose to be singletons and the last coalition is formed by the last (the first integer previous to) $r^{\max }(n)$ players. (For more details, see Bloch 1996, p. 122). Note that $r^{\max }(n)$ is increasing in $n$. 
When the moral hazard problem is not very severe $(a / c \geq n+2)$, we know (by Proposition 2ii) that all singletons cannot be an equilibrium outcome. Proposition 4 states that, in this case, the equilibrium coalition structure is never more deconcentrated than without moral hazard. In fact, for some values of the parameters it is strictly more concentrated. ${ }^{9}$ This is the case even if moral hazard creates an inefficiency that grows with the size of the coalition and one would expect that partners would then try to avoid it by forming smaller groups than in the benchmark case. The intuition is related to the sequential nature of the game. Consider first the case without moral hazard. In equilibrium, the first players freeride on the last players by standing alone, until the point where the deciding player faces the alternative of inducing an all-singleton outcome or to joining the remaining players in a single coalition. With moral hazard this free-riding by the first players is more difficult. The last players find forming a big coalition very harmful and they are only ready to group into small coalitions in concentrated markets. It may be the case that they are willing to form a partnership only in environments where the previous players have also joined coalitions. Anticipating this reaction, the first players have less incentives to split off. This effect may lead to a more concentrated structure than the equilibrium outcome without moral hazard.

In the following Corollary, we summarize the most relevant feature of the equilibrium outcomes.

Corollary 1.- For a given $n$, the equilibrium coalition structure with moral hazard is either the most deconcentrated outcome (all singletons) or one structure containing a number of coalitions lower than or equal to the number of equilibrium coalitions without moral hazard.

In our model of Cournot competition with moral hazard, we cannot go further and provide and explicit expression for the size and composition of partnerships at equilibrium as a function of the parameters of the model $(a, c, n)$. However, we can compute the equilibrium outcome for given values of $n$. In Table 1 we present the equilibrium outcome

9 Our measure of market concentration is just the inverse of the number of partnerships. 
for $n=18$. The last row indicates the equilibrium outcome in the benchmark case (without moral hazard).

[Insert Table 1]

From Table 1 we see that, unless $c$ is high enough $\left(\frac{a}{c}<20\right)$ so that the only sustainable partition is all partners as singletons, moral hazard makes the equilibrium market structure either similar to or more concentrated than the benchmark case. As the game is sequential, the first players know that moral hazard would prevent the last players from forming large groups; the threat of a less concentrated market structure makes the first players choose not to stand alone. In fact, as this example illustrates, this threat induces the first player to find it optimal to form a coalition with some other players in order to leave a low enough number of players in the game so that it is profitable for them to collude.

\section{4.- On the robustness of the results and the effects of moral hazard under other stability concepts}

We have shown the effects of moral hazard in a finite choice-of-sizes game. The interest of this game comes from the following equivalence result by Bloch (1996) and Ray and Vohra (1999): In a symmetric offer and counteroffer infinite game $\Gamma$, any symmetric stationary perfect equilibrium coalition structure can be reached as the outcome of a finite game of choice of coalition sizes $\Delta$. Moreover, if the equilibrium coalition structure of the finite game has the property that players' payoffs are non-increasing in the order in which coalitions are formed, then any equilibrium outcome of the game of choice of coalition sizes $\Delta$ can be obtained as a symmetric stationary equilibrium coalition structure of the sequential infinite game of coalition formation $\Gamma$.

However, as the example in Table 1 illustrates (for $n=18$ ), in our model there are values of the parameters $(a, c, n)$ such that the payoffs of the first players are lower than the profits of the last ones (that is for $a / c \in[27,28.2)$ ). For those parameter values there is no symmetric stationary equilibrium of the infinite game; nevertheless, in those cases the choice-of-sizes game still identifies an outcome that, as we show in the Appendix, corresponds to an asymmetric stationary equilibrium in the infinite game $\Gamma$. The idea for 
constructing such an equilibrium of $\Gamma$ is simple. When players' payoffs are increasing in the order in which coalitions are formed, then there is no symmetric stationary equilibrium since any player will prefer to deviate (to reject the offer and to make an unaccepted offer) in order to be in the last coalition (or the one having the highest payoffs). The asymmetric equilibrium presented in the Appendix is based on strategies such that a set of players agree to form the first coalition in order to avoid an infinite playing of the game.

Even when the property holds (and the equivalence between the finite choice-of-sizes game $\Delta$ and the infinite game $\Gamma$ holds) the question remains as to whether moral hazard would have the same effect (increasing market concentration) had we used a different game. To analyze this point we have looked into the effect of moral hazard in some other coalition formation games and different stability notions.

A first observation is that the effect of moral hazard pointed out in this paper will not be present unless the game is somehow sequential. Moral hazard makes unilateral defections from a coalition more profitable so that, if the game with payoffs (4) is one-shot (see Yi and Shin (1995) exclusive membership game), sustaining a concentrated coalition structure (as a NE or a CPNE) is made harder when moral hazard is introduced.

We briefly consider here the effect of moral hazard using another sequential solution concept. Ray and Vohra (1997) introduced the notion of equilibrium binding agreements. A coalitional structure is an equilibrium binding agreement if there is no profitable deviation for any set of players, but deviators take into account what happens after the deviation, in particular, the strategies of the complement are not taken as given (the complementary coalitions may break up). Ray and Vohra define a profitable deviation (from one coalitional structure to another) for a set of players as a deviation satisfying the following conditions: (i) the final structure is an equilibrium binding agreement, (ii) the deviating players must be a sub-coalition of one of the initial coalitions, (iii) the deviating set of players must gain from this move, and (iv) the other deviators fear a worse outcome if they do not move. ${ }^{10}$ In this framework, under moral hazard the threat of inducing a deviation into a very 
competitive market may induce a more concentrated structure (as compared to stable structures without moral hazard). To discuss the effect of moral hazard, we present the results for $n=6$ in Table 2 .

\section{[Insert Table 2]}

For $n=6$, with no moral hazard, the most concentrated stable outcomes are triopolies. With moral hazard, some of these triopolies are unstable when moral hazard is strong enough. As in Section 3, for $\frac{a}{c}$ high enough we have the same result as without moral hazard. However, when the moral hazard problem is important more concentrated structures are stable, because then the asymmetric triopoly structure $\{1,2,3\},\{4,5\},\{6\}$ is no longer a stable outcome; the larger coalition is suffering from an important inefficiency and the deviation to all singletons is profitable. This effect makes some duopoly coalition structures stable under moral hazard. For example, for $\frac{a}{c} \in[57,106[$, if a subset of players deviates from $\{1,2,3$, $4,5\},\{6\}$ they will not reach a triopoly, but the stable outcome $(\{1\},\{2\},\{3\},\{4\},\{5\},\{6\})$, where competition is very strong.

\section{5.- Industry Efficiency}

In this section we briefly discuss the efficiency of the outcome of the sequential process of partnership formation described above. An efficient partnership configuration is defined here as one that (given that partnerships compete in the market in the second stage) yields the highest industry profits among all the market structures.

With no moral hazard the efficient outcome is always the grand coalition, where industry profits are maximized. The equilibrium market structure may be inefficient since duopoly or even less concentrated market structures obtain in equilibrium. Moral hazard adds an element of inefficiency to large firms which can make the grand coalition inefficient. ${ }^{11}$

11 Another way of understanding this point is that without moral hazard the grand coalition will decide to form a single firm, while with moral hazard the grand coalition may decide to set up more than one firm. 
Remark 1.- With moral hazard, the equilibrium market structure may be efficient.

We present an example where this happens to be the case. Let us consider the example of $n$ $=18$. It can be easily checked that for some parameter configurations equilibrium is an efficient outcome. More precisely, we find values of $c$ for which the outcome maximizes industry profits, $\frac{a}{c} \in[46,84)$, which is never the case in the absence of moral hazard. When $c$ is very low $\left(\frac{a}{c} \geq 84\right)$ too many coalitions are formed. The reason for this inefficiency is the usual free-riding problem already present in the benchmark case. ${ }^{12}$

\section{6.- Concluding comments}

Previous work on endogenous coalition formation with externalities (spillovers) is based on the assumption that the outcome of a coalition should be efficient for that coalition. Even though there is interaction among the players at two levels: within each coalition and between coalitions, the first aspect has been neglected in the non-cooperative models of coalition formation. The only aspect of that interaction that has been taken into account is the definition of the sharing rule agreed upon by the coalition members. However, there is a great deal of literature dealing with the inefficiencies that arise within groups due to imperfect effort observability, the lack of incentives to cooperate, and the lack of commitment capacity. This is the aim of our analysis: to provide some insight on the effects that the internal organization of coalitions may have on the equilibrium coalition structure.

In a model with ex-ante identical players, we analyze the non-cooperative process of coalition formation (this process is formalized as in Bloch, 1995, 1996), with a moral hazard problem within coalitions, and we study the equilibrium number and size of coalitions. As compared to the case where members in a coalition fully cooperate, we show that when moral hazard within coalitions is high enough, no coalition will form. However, and this result is more surprising, when moral hazard is not too severe the coalition

12 For low values of $a / c$ the comparison is more artificial since the production of the firms in the efficient configuration may not be interior. 
structure will be either similar to or more concentrated than without moral hazard (that is, a lower number of partnerships in a subgame perfect equilibrium).

To emphasize some of the aspects of the relationship between the incentive problems within partnerships, market competition, and stability of coalitional configurations, we have ignored other important elements of partnership organization. The firm may embody interactions (joint task responsibilities, or specialization) between agents that increase the productivity of a worker when they work in a large team. Another central aspect of the model is that we obtain our results under the assumption that a coalition splits gross profits equally. Under moral hazard the sharing rule is crucial, not only in deciding whether to join a coalition or not, but also because it determines the partners' effort decision and consequently, gross profits. The lower the share of a partner, the more opportunistically she will behave. However, equal sharing is the most natural sharing rule in a symmetric framework and represents the spirit of what it is understood as a partnership. 


\section{References}

Belleflamme, P., 2000, "Stable Coalition Structures with Open Membership and Asymmetric Firms", Games and Economic Behavior 30, 1-21.

Bloch, F., 1995, "Endogenous Structures of Associations in Oligopolies," The Rand Journal of Economics 26, 537-555.

Bloch, F., 1996, "Sequential Formation of Coalitions in Games with Externalities and Fixed Payoff Division," Games and Economic Behaviour 14, 90-123.

Farrell J. and S. Scotchmer, 1988, "Partnerships," Quarterly Journal of Economics, 279297.

Hart, S., 1974, "Formation of Cartels in Large Markets," Journal of Economic Theory 7, 453-466.

Hart, S. and M. Kurz, 1983, "Endogenous Formation of Coalitions," Econometrica 51, 1047-1064.

Holmström B., 1982, “Moral Hazard in Teams,” The Bell Journal of Economics, 324-340.

Macho-Stadler, I. and D. Pérez-Castrillo, 1997, An Introduction to the Economics of Information: Incentives and Contracts, Oxford University Press.

Montero, M., 1999, "Noncooperative Bargaining in Partition Function Games", mimeo CentER.

Ray, D. and R. Vohra, 1997, "Equilibrium Binding Agreements," Journal of Economic Theory 73(1), 30-78.

Ray, D. and R. Vohra, 1999, "A Theory of Endogenous Coalition Structure”, Games and Economic Behavior 26(2), 286-336.

Salant, S. W., Switzer, S. and Reynolds, R. J., 1983, "Losses from Horizontal Merger: The Effects of an Exogenous Change in Industry Structure on Cournot-Nash Equilibrium," Quarterly Journal of Economics 98, 185-199.

Yi, S.-S., 1997, "Stable Coalition Structures with Externalities," Games and Economic Behavior 20(2), 201-37.

Yi, S.-S. and Shin, H., 1995, "Endogenous Formation of Coalitions in Oligopoly," Dartmouth College Working Paper 95-2. 


\section{Appendix}

Proof of Proposition 1.- From (4) we can deduce that $\pi(r, s)>0$ if and only if $\frac{a}{c}+n-s-$ $s r>0$, i.e., $s<\frac{a / c+n}{r+1}$. (Note that $\pi(r, 1)>0$ for any $r$ ). Summing up for the $r$ coalitions we have that $n<r \frac{a / c+n}{r+1}$ is a necessary condition for $\pi(r, s)>0$, which can be rewritten as $\frac{n}{r}<\frac{a}{c}$.

If $\frac{n}{r}>\frac{a}{c}$ for any $r<n$, then the necessary condition for all the coalitions to have positive profits does not hold. Thus, $1<\frac{a}{c}<\frac{n}{n-1}$ is a sufficient condition for the partition $r=n$ to be the only SPE partition.

Q.E.D.

Proof of Lemma 1.- Player $(n-x+1)$ deciding in subgame $(h, x)$ the optimal size $s$, under the assumption that the rest of the players will remain singletons, solves:

$$
\max _{1 \leq s \leq x}\left\{\frac{\left[\frac{a}{c}+n-(h+x-s+2)\right]\left[\frac{a}{c}+n-s(h+x-s+2)\right]}{s(h+x-s+2)^{2}}\right\}
$$

The first-order derivative has the same sign as:

$G(s) \equiv-\left(\frac{a}{c}+n-(h+x+2)\right)(h+x+2-3 s)-s^{2}(h+x-s)$.

For $s=1, G$ is negative (and the objective function is decreasing). Now, given that $G^{\prime}(s)=$ $3\left(\frac{a}{c}+n-(h+x+2)\right)-2 s(h+x)+3 s^{2}$, and $G^{\prime \prime}(s)=-2(h+x)+6 s$, we know that $G^{\prime}(s)$ has a minimum at $s=\frac{h+x}{3}$. At this point: $G^{\prime}\left(\frac{h+x}{3}\right)=9\left[\frac{a}{c}+n-(h+x+2)\right]-$ $\frac{(h+x)^{2}}{3}$. Consequently: 
(a) If $9\left(\frac{a}{c}+n-(h+x+2)\right)-(h+x)^{2}>0, G^{\prime}(s)$ is always positive, and $G(s)$ is always increasing.

(b) If $9\left(\frac{a}{c}+n-(h+x+2)\right)-(h+x)^{2}<0$, then $G^{\prime}\left(\frac{h+x}{3}\right)<0$ and $G^{\prime}(s)$ has two zero values (possibly outside the range of our parameter selection). $G^{\prime}(s)=0$ for $s=\frac{(h+x) \pm\left[(h+x)^{2}-9\left[\frac{a}{c}+n-(h+x+2)\right]\right]^{\frac{1}{2}}}{3}$. At the lowest of these values $G(s)$ is negative.

In both cases, $G^{\prime}(s)$ goes from a negative value at $s=1$, to a (possibly) positive expression. Hence, the objective function is either decreasing in $s$ or convex in $s$. In any case the solution to the maximization problem will be reached at a corner.

Q.E.D.

\section{Proof of Lemma 2.-}

2 (i) By the definition of $g(h, x)$ :

$$
\begin{aligned}
g(h, x) & =\frac{1}{x}\left(\frac{a+c n-(h+2) c}{h+2}\right)\left(\frac{a+c n-x(h+2) c}{h+2}\right)-\left(\frac{a+c n-(h+x+1) c}{h+x+1}\right)^{2}= \\
& =(a+c n)^{2}\left[\frac{(h+x+1)^{2}-x(h+2)^{2}}{x(h+x+1)^{2}(h+2)^{2}}\right]- \\
& -(a+c n) c\left[\frac{(1+x)(h+x+1)^{2}(h+2)-2 x(h+2)^{2}(h+x+1)}{x(h+x+1)^{2}(h+2)^{2}}\right]
\end{aligned}
$$

Then: $\operatorname{sign}[g(h, x)]=\operatorname{sign}\left[(a+c n)\left[(h+\mathrm{x}+1)^{2}-x(h+2)^{2}\right]-c\left[(1+x)(h+2)(h+x+1)^{2}-2 x\right.\right.$ $\left.\left.(h+2)^{2}(h+x+1)\right]\right]=\operatorname{sign}\left[(a+c n)\left(x-(h+1)^{2}\right)-c(h+2)\left(x^{2}-(h+1)^{2}\right)\right] . \quad$ Q.E.D.

2 (ii) From Lemma 2 (i), if $x-(h+1)^{2}<0$ and $x^{2}-(h+1)^{2} \geq 0$, then $g(h, x)<0$. If $x-$ $(h+1)^{2}<0$ and $x^{2}-(h+1)^{2} \leq 0$, it can be checked that $g(h, x)<0$. Thus, $x-(h+1)^{2}>0$ is a necessary condition for $g(h, x) \geq 0$.

Q.E.D. 
2 (iii) From Lemma 2 (ii), assume that $x \leq(h+1)^{2}$, and hence $g(h, x)<0$. Let us consider any subgame $\left(h^{\prime}, x^{\prime}\right)$ of $(h, x)$. Then $x^{\prime}<\left(h^{\prime}+1\right)^{2}$ given that $h^{\prime}>h$ and $x^{\prime}<x$. Therefore, $g\left(h^{\prime}, x^{\prime}\right)<0$.

Q.E.D.

Proof of Proposition 3.- We define a function $\phi(h, x)=\frac{(h+2)\left[x^{2}-(h+1)^{2}\right]}{x-(h+1)^{2}}$, in the interval $n-h \geq x>(h+1)^{2}$. Note that the argument that $\max _{x} \mathrm{~g}(h, x)$ is the same as the argument that $\min _{x} \phi(h, x)$. Moreover, $\frac{a}{c} \geq \phi(h, x)-n$ is equivalent to $g(h, x) \geq 0$; and $\frac{a}{c}<$ $\min _{x} \phi(h, x)-n$ is equivalent to $\max _{x} g(h, x)<0$. Finally, it is easy to check that $\phi(h, x)$ is an increasing function of $h$ and it is convex in $x$. We proceed in several steps.

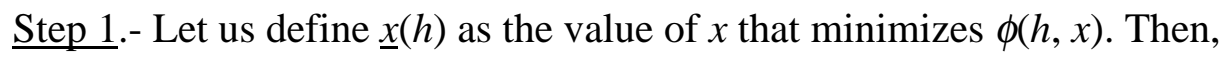

$$
\underline{x}(h)=(h+1)\left(h+1+\sqrt{h^{2}+2 h}\right) .
$$

Moreover, $\underline{x}(h)$ is increasing in $h$.

Proof.- From the second-order derivative, it is easy to check that (in the relevant range) $\phi(x, h)$ is convex in $x$. From the first-order derivative of $\phi(x, h)$ with respect to $x$ we can conclude that the minimum is reached at the solution of the following equation:

$$
x^{2}-2 x(h+1)^{2}+(h+1)^{2}=0 \text {. }
$$

The solutions to this equation are:

$$
x(h)=(h+1)((h+1) \pm \sqrt{h(h+2)}) .
$$

Taking into account that we restrict our attention to $x>(h+1)^{2}$, we can conclude that the minimum is reached at $\underline{x}(h)=(h+1)((h+1)+\sqrt{h(h+2)})$. This function is increasing in $h$.

In fact, the solution in the set of natural numbers is an integer after or before $\underline{x}(h)$. We will show in due course that this is not a problem when looking for necessary conditions.

Step 2.- For $r<n$, a necessary condition for an $r$-poly to be a stable coalition configuration is that $g(r-1, x) \geq 0$, for some $x \leq n-r+1$. 
Proof.- For an $r$-poly to be stable, it is necessary that $(r-1)$ coalitions must be formed, there must then be at least two remaining players and the player taking the decision must choose to form a single coalition with all of them, or it must already be player $n$ 's turn. We are going to show that a necessary condition for the above property to hold is that there is at least one $x, x \leq n-r+1$, for which $g(r-1, x) \geq 0$.

We do the proof by contradiction, i.e., we assume that $g(r-1, x)<0$, for all $x \leq n-r+1$, and we show that:

(a) if $(r-1)$ coalitions have been formed and there are at least two players, the deciding player will never form a single coalition,

(b) a situation in which $(r-1)$ coalitions have been formed when player $n$ is called to play cannot be part of an SPE of the game.

(a) Suppose that $(r-1)$ coalitions are formed and there are $x \geq 2$ remaining players. Since $g(r-1, x)<0$, player $n-x+1$ (who is called to play) prefers the remaining players to split off rather than forming a single coalition. We now claim that for player $n-x+1$ staying alone (i.e., choosing $s_{r}=1$ ) is better than forming a single coalition (choosing $s_{r}=x$ ). Indeed, from this player's point of view, the most damaging strategy by the subsequent players is for them to remain singletons (if any subgroup decided to form a coalition, the profits of player $n-x+1$ would increase). Even in this case, profits for player $n-x+1$ are higher than if she decides to form a single coalition.

(b) Suppose that $(r-1)$ coalitions are formed before player $n$. Now, take the last coalition formed containing $x \geq 2$ players (note that we can put forward this argument because $r<$ $n)$. For the player that forms this coalition, it must be the case that $\pi(r, x) \geq \pi(r+x, 1)$, i.e., $g(r-1, x) \geq 0$, which contradicts our assumption.

Step 3.- For $r<n$, a necessary condition for an $r$-poly to be a stable coalition configuration is:

i) $\frac{a}{c}+n \geq \frac{(r+1)(n+1)(n-2 r+1)}{\left(n+1-r-r^{2}\right)}$, if $\underline{x}(r-1)>n-r+1$, or 
ii) $\frac{a}{c}+n \geq 2 r(r+1)\left(r+\sqrt{r^{2}-1}\right)$, if $\underline{x}(r-1) \leq n-r+1$.

Proof.- First note that for any $1 \leq x \leq r^{2}$, we always have that $g(r-1, x)<0$. By Step 2, a necessary condition for an $r$-poly to be a stable coalition configuration is that $g(r-1, x) \geq 0$ for some $x \leq n-r+1$, i.e., $g(r-1, x) \geq 0$ for some $r^{2}<x \leq n-r+1$. This condition is equivalent to: $\frac{a}{c}+n \geq \operatorname{Min} \phi(r-1, x)$, for $r^{2}<x \leq n-r+1$.

i) If $n-r+1<\underline{x}(r-1)$ then the minimum is reached at $x=n-r+1$. Then, a necessary condition for $r$-poly to be a stable coalition structure is $\frac{a}{c}+n \geq \phi(r-1, n-r+1)=$ $\frac{(r+1)(n+1)(n-2 r+1)}{\left(n+1-r-r^{2}\right)}$.

ii) If $n-r+1 \geq \underline{x}(r-1)$ then the minimum is reached at $\underline{x}(r-1)$. Then, a necessary condition for $r$-poly to be an stable coalition configuration is $\frac{a}{c}+n \geq \phi(r-1, \underline{x}(r-1))=$ $2 r(r+1)\left(r+\sqrt{r^{2}-1}\right) .^{13}$

Step 4.- $\left.\left.\left.\left.[g(r-1, x) \leq 0, \forall x \in] r^{2}, n-r+1\right]\right] \Rightarrow[g(r, x)<0, \forall x \in](r+1)^{2}, n-r\right]\right]$.

Proof.- The previous implication is equivalent to

13 As we have already mentioned, $\underline{x}(r-1)$ need not be a natural number. But $\left.\left.\left.\left.\{\operatorname{Min} \phi(r-1, x), \forall x \in] r^{2}, n-r+1\right], x \in R\right\} \leq\{\operatorname{Min} \phi(r-1, x), \forall x \in] r^{2}, n-r+1\right], x \in N\right\}$

Therefore, $\left.\left.\frac{a}{c}+n \geq\{\operatorname{Min} \phi(r-1, x), \forall x \in] r^{2}, n-r+1\right], x \in R\right\}=\phi(r-1, \quad \underline{x}(r-1))$ is also a (weaker) necessary condition for an $r$-poly to be a stable coalition structure. 


$$
\begin{aligned}
& \left.\left.\left[\frac{a}{c}+n<\{\operatorname{Min} \phi(r-1, x), \forall x \in] r^{2}, n-r+1\right]\right\}\right] \Rightarrow \\
& \left.\left.\left[\frac{a}{c}+n<\{\operatorname{Min} \phi(r, x), \forall x \in](r+1)^{2}, n-r\right]\right\}\right] .
\end{aligned}
$$

This in turn is equivalent to:

$\left.\left.\left.\left.[\operatorname{Min} \phi(r-1, x), \forall x] r^{2}, n-r+1\right]\right]<[\operatorname{Min} \phi(r, x), \forall x](r+1)^{2}, n-r\right]\right]$. This condition holds since: a) $\phi$ is increasing in $h$, and b) $\phi(r-1, n-r+1)<\operatorname{Min} \phi(r, n-r) . \quad$ Q.E.D.

\section{An asymmetric stationary equilibrium of the infinite game $\Gamma$}

Let us denote by $S_{1}, S_{2}, \ldots, S_{h}$ the coalitions already formed at subgame $(h, x) ; X=$ $M \cup_{k=1}^{h} S_{k}$, the players not allocated to a coalition yet, and $S\left(S_{1}, S_{2}, \ldots, S_{\mathrm{h}}, X\right)$ is the ongoing proposal. A strategy for a player must specify an answer to any proposal made to her if she is not the proposer, and a proposal when she is. Formally,

$$
\begin{array}{ll}
\sigma_{i}\left(S_{1}, S_{2}, \ldots, S_{h}, X\right) \in\{\text { Yes, NO }\} & \text { if } S\left(S_{1}, S_{2}, \ldots, S_{h}, X\right) \neq \varnothing \\
\sigma_{i}\left(S_{1}, S_{2}, \ldots, S_{h}, X\right) \in\{S \subset X, i \in S\} & \text { if } S\left(S_{1}, S_{2}, \ldots, S_{h}, X\right)=\varnothing
\end{array}
$$

When $S\left(S_{1}, S_{2}, \ldots, S_{h}, X\right) \neq \varnothing$, player $i$ is a respondent to the offer by some other player; when $S\left(S_{1}, S_{2}, \ldots, S_{h}, X\right)=\varnothing$, it is player $i$ 's turn to make an offer. If the strategy's prescription at any subgame depends only on the number of coalitions previously formed and the number of players still in the game, we simply write $\sigma_{i}(h, x)$.

For $n=18$ and $\frac{a}{c} \in\left[27,28.2\left[\right.\right.$, let $N_{1}=\{1,2, \ldots, 10\}$ and $N_{2}=\{11, \ldots, 18\}$. Consider the strategy profile for game $\Gamma$ shown in Table 4 . It is easy to check that the equilibrium is not symmetric since players in $N_{1}$ and $N_{2}$ follow different strategies; it is stationary because players' strategies depend only on $(h, x)$.

\section{[Insert Table 3]}

Given the one-stage deviation principle for infinite games, in order to check that the strategies lead to a subgame perfect equilibrium it is sufficient to check that there is no ( $h$, 
$x$ ) where a player can gain by deviating from the action prescribed by the strategy for that history and conforming to the initial strategy thereafter. The one-stage deviation principle holds whenever the infinite game is continuous at infinity, i.e., the events in the distant future are relatively unimportant, which is the case in our infinite game if future payments are discounted.

In order to check the equilibrium, note first that for $n=18$ :

$$
\frac{a}{c} \geq 27 \Leftrightarrow g(1,8) \geq 0 \text { and } \frac{a}{c}<28.2 \Leftrightarrow g(1,9)<0 .
$$

In this interval, $g(2, x)$ is always negative. Then, once two coalitions have been formed, the best response for any remaining player is to stay alone, as the strategy in Table 3 states. When one coalition has been formed, only coalitions sized 8 or less are profitable, as prescribed by the strategy. The only asymmetry is in the prescribed actions for different players when no coalition has been formed. Ten players, from 1 to 10 , are ready to form a 10 player coalition, while the others are not. For the first players, there is no gain in deviating to any other action (that will induce too much competition) since the last players will only join a coalition if the first players have done so before.

Q.E.D. 


\begin{tabular}{|c|c|c|}
\hline$\frac{\text { Demand }}{\text { and cost conditions }}$ & $\frac{\text { Equilibrium market }}{\underline{\text { structure }}}$ & $\underline{\text { Partition }}$ \\
\hline$\frac{a}{c} \in(1,20)$ & singletons & {$[\{1\},\{2\} \ldots,\{18\}]$} \\
\hline$\frac{a}{c} \in[20,27)$ & monopoly & {$[\{1,2, \ldots ., 18\}]$} \\
\hline$\frac{a}{c} \in[27,28.2)$ & duopoly & {$[\{1, \ldots, 10\},\{11, \ldots, 18\}]$} \\
\hline$\frac{a}{c} \in[28.2,30)$ & duopoly & {$[\{1, \ldots, 9\},\{10, \ldots, 18\}]$} \\
\hline$\frac{a}{c} \in[30,32.143)$ & duopoly & {$[\{1, \ldots, 8\},\{9, \ldots, 18\}]$} \\
\hline$\frac{a}{c} \in[32.143,34.5)$ & duopoly & {$[\{1, \ldots, 7\},\{8, \ldots, 18\}]$} \\
\hline$\frac{a}{c} \in[34.5,37)$ & duopoly & {$[\{1, \ldots, 6\},\{7, \ldots, 18\}]$} \\
\hline$\frac{a}{c} \in[37,39.6)$ & duopoly & {$[\{1, \ldots, 5\},\{6, \ldots, 18\}]$} \\
\hline$\frac{a}{c} \in[39.6,42.27)$ & duopoly & {$[\{1,2,3,4\},\{5, \ldots, 18\}]$} \\
\hline$\frac{a}{c} \in[42.27,45)$ & duopoly & {$[\{1,2,3\},\{4, \ldots, 18\}]$} \\
\hline$\frac{a}{c} \in[45,47.77)$ & duopoly & {$[\{1,2\},\{3, \ldots, 18\}]$} \\
\hline$\frac{a}{c} \in[47.77,123.14)$ & duopoly & {$[\{1\},\{2, \ldots, 18\}]$} \\
\hline$\frac{a}{c} \in[123.14, \infty)$ & triopoly & {$[\{1\},\{2\},\{3, \ldots, 18\}]$} \\
\hline
\end{tabular}

Table 1. Equilibrium partnership configuration for $n=18$ 


\begin{tabular}{|c|c|c|c|}
\hline Coalition structure & $\frac{\text { Conditions for deviation }}{\text { (with } \mathrm{MH} \text { ) }}$ & $\begin{array}{l}\text { Stability without } \\
\text { Moral Hazard }\end{array}$ & $\begin{array}{l}\text { Stability with } \\
\text { Moral Hazard }\end{array}$ \\
\hline$\{1\},\{2\},\{3\},\{4\},\{5\},\{6\}$ & (none) & By definition & By definition \\
\hline$\{1,2\},\{3\},\{4\},\{5\},\{6\}$ & $\begin{array}{l}\text { for any } a / c, \text { to } \\
\{1\},\{2\},\{3\},\{4\},\{5\},\{6\}\end{array}$ & No & No \\
\hline$\{1,2,3\},\{4\},\{5\},\{6\}$ & $\begin{array}{l}\text { for any } a / c, \text { to } \\
\{1\},\{2\},\{3\},\{4\},\{5\},\{6\}\end{array}$ & No & No \\
\hline$\{1,2\},\{3,4\},\{5\},\{6\}$ & $\begin{array}{l}\text { for any } a / c, \text { to } \\
\{1\},\{2\},\{3\},\{4\},\{5\},\{6\}\end{array}$ & No & No \\
\hline$\{1,2,3,4\},\{5\},\{6\}$ & $\begin{array}{l}\text { for any } a / c, \text { to } \\
\{1\},\{2\},\{3\},\{4\},\{5\},\{6\}\end{array}$ & No & No \\
\hline$\{1,2,3\},\{4,5\},\{6\}$ & $\begin{array}{l}\text { for } a / c<160, \text { to } \\
\{1\},\{2\},\{3\},\{4\},\{5\},\{6\}\end{array}$ & Yes & $\begin{array}{l}\text { Yes, for } \\
a / c \geq 106\end{array}$ \\
\hline$\{1,2\},\{3,4\},\{5,6\}$ & $\begin{array}{l}\text { for } a / c<2.235, \text { to } \\
\{1\},\{2\},\{3\},\{4\},\{5\},\{6\}\end{array}$ & Yes & $\begin{array}{l}\text { Yes, for } \\
a / c \geq 2.235\end{array}$ \\
\hline$\{1,2,3,4,5\},\{6\}$ & $\begin{array}{l}\text { for } a / c \geq 106, \text { to } \\
\{1,2,3\},\{4,5\},\{6\} \\
\text { for } a / c<57, \text { to } \\
\{1\},\{2\},\{3\},\{4\},\{5\},\{6\}\end{array}$ & No & $\begin{array}{l}\text { Yes, for } \\
57 \leq a / c<106\end{array}$ \\
\hline$\{1,2,3,4\},\{5,6\}$ & $\begin{array}{l}\text { for } a / c \geq 2.235, \text { to } \\
\{1,2\},\{3,4\},\{5,6\} \\
\text { for } a / c<2.235, \text { to } \\
\{1\},\{2\},\{3\},\{4\},\{5\},\{6\}\end{array}$ & No & No \\
\hline$\{1,2,3\},\{4,5,6\}$ & $\begin{array}{l}\text { for } a / c<3.55, \text { to } \\
\{1\},\{2\},\{3\},\{4\},\{5\},\{6\} \\
\text { for } a / c \geq 106, \text { to } \\
\{1,2,3\},\{4,5\},\{6\}\end{array}$ & No & $\begin{array}{l}\text { Yes, for } \\
3.55 \leq a / c<106\end{array}$ \\
\hline$\{1,2,3,4,5,6\}$ & $\begin{array}{l}\text { for } a / c<106, \text { to } \\
\{1,2,3\},\{4,5\},\{6\} \\
\text { for } a / c \geq 106, \text { to } \\
\{1,2,3,4,5\},\{6\}\end{array}$ & No & No \\
\hline
\end{tabular}

Table 2. Equilibrium Binding Agreements (Ray and Vohra) for $n=6$ 


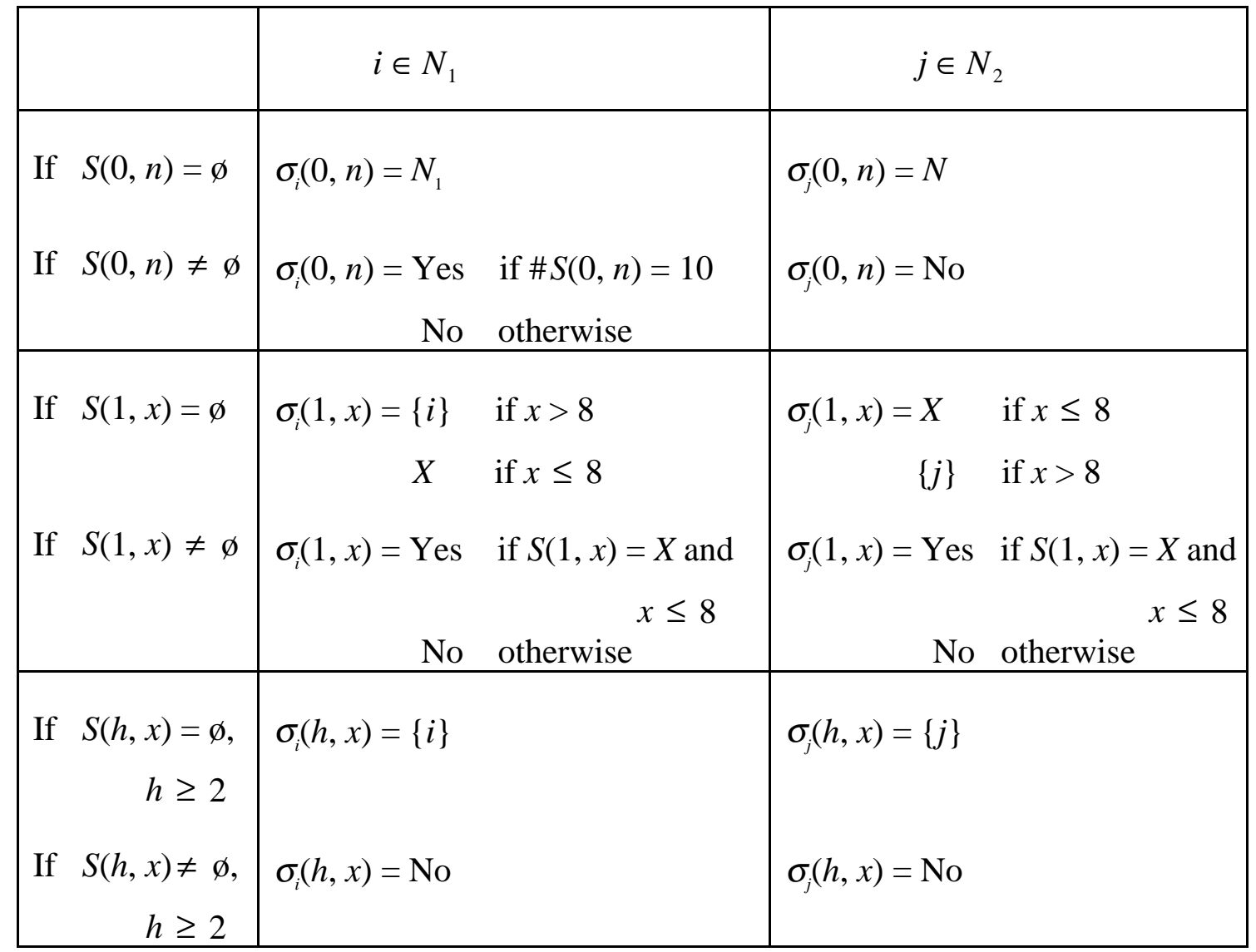

Table 3. Strategies supporting the stationary and asymmetric equilibrium of $\Gamma$ 